\title{
Effect of Drug Loading on the Physicochemical Properties and Stability of Cationic Lipid-based Plasmid DNA Complexes
}

\author{
Ui-Hyeon Jeong ${ }^{1, *}$, Ji-Hye Jung ${ }^{1, *}$, Enkhzaya Davaa ${ }^{1}$, Se-Jin Park ${ }^{1}$, Chang-Seon Myung ${ }^{2,3}$ and Jeong-Sook Park $^{1,3 \dagger}$ \\ ${ }^{1}$ Department of Physical Pharmacy, College of Pharmacy, Chungnam National University, Daejeon 305-764, Korea \\ ${ }^{2}$ Department of Pharmacology, College of Pharmacy, Chungnam National University, Daejeon 305-764, Korea \\ 3 Institute of Drug Research and Development, Chungnam National University, Daejeon 305-764, Korea
}

(Received September 21, $2009 \cdot$ Revised September 24, 2009 - Accepted September 28, 2009)

\begin{abstract}
Recently, co-delivery of drug and gene has been attempted for higher therapeutic effects of anticancer agents. In this study, cationic liposomes were prepared using 1,2-dioleoyl-3-trimethylammoniopropane (DOTAP) as a cationic lipid to investigate the effect of drug loading on the physicochemical characteristics of cationic liposomes/DNA complexes. The complex formation between cationic liposomes and negatively charged plasmid DNA was confirmed and the protection from DNase was observed. Particle size of complexes was reduced not by drug loading, but by the increased ratio of cationic lipid to plasmid DNA. Meanwhile, zeta potential of complex was increased by the addition of cationic liposomes to complexes and the effect of drug loading on the zeta potential was not much higher than on particle size. Gel retardation of complexes was indicated when the complexation weight ratios of cationic lipid to plasmid DNA were higher than 24:1 for drug free complexes and 20:1 for drug loaded ones, respectively. Agarose gel retardation showed the similar complexation between plasmid DNA and drug free liposomes or drug loaded liposomes. Both complexes protected plasmid DNA from DNase independent of complexation temperature. From the results, drug loading may affect not the complex formation of cationic liposomes and plasmid DNA, but the particle size of complex.
\end{abstract}

Key words - cationic liposomes, drug loading, stability, DNase

The object of gene therapy is to deliver a therapeutic gene to selected cells where proper gene expression can be achieved. ${ }^{1)}$ An ideal gene delivery vector requires 3 major criteria: (1) it should protect the transgene against degradation by nucleases and deliver the transgene into the nuclei of target cells, (2) it should be non-toxic and non-immunogenic, and (3) it should be easily prepared for the long-term gene expression. ${ }^{2)}$ Currently investigated vectors are classified as viral and non-viral. Viral vectors are able to mediate gene transfer with high efficiency, but immune response and inflammation in clinical trials have raised serious safety concern about commonly used viral vectors. ${ }^{1)}$ Non-viral gene delivery vectors attract attention due to their non-immunogenic properties as opposed to their viral counterparts.

Until now combination drug therapies of anticancer agents have been attempted to overcome drug resistance in clinical applications, ${ }^{3-5)}$ but success has been limited. Currently it is suggested that drug resistance is mainly caused by malfunction of genes due to chromosomal alterations in cancer cells. ${ }^{6,7)}$ As

\footnotetext{
*These authors contributed equally to this work

†본 논문에 관한 문의는 이 저자에게로

Tel : 042)821-5932, E-mail : eicosa@cnu.ac.kr

DOI : 10.4333/KPS.2009.39.5.339
}

a result, correcting malfunctioned genes through siRNA or anticancer gene delivery has been attempted to sensitize cancer cells towards anticancer drugs. ${ }^{8-10)}$ In addition, pretreatment of cells with anticancer drugs such as paclitaxel has been also reported to enhance transgene expression of reporter genes. ${ }^{11,12)}$ Moreover, the simultaneous delivery of paclitaxel and IL-12 encoding plasmid using cationic micelles (or nanoparticles) was demonstrated to achieve a synergistic effect in suppressing cancer growth in a mouse breast cancer model, and co-delivery of the same drug and Bcl-2 targeted siRNA was also found to sensitize MDA-MB-231 human breast cancer cell line to paclitaxel. ${ }^{13,14)}$ Moreover, it was reported that synergistic therapeutic effect was obtained by cationic micelles for co-delivery of drug and gene. ${ }^{15)}$ Co-delivery of drug and gene in the same vehicle not only can improve compliance due to the reduced number of administrations, but can also achieve a synergistic therapeutic effect because both drug and gene can be delivered to the same cells or tissues.

In this study, we prepared cationic liposomes using DOTAP as a cationic lipid, DOPE and cholesterol as helper lipids to deliver drug and plasmid DNA simultaneously. Then, we investigated the effect of drug loading on the physicochemical properties and stability of complexes to figure out the feasibility of the co-delivery of drug and DNA. 


\section{Experimental}

\section{Materials}

1,2-dioleoyl-3-trimethylammoniopropane (DOTAP), 1,2dioleoyl-sn-glycerol-phosphoethanolamine (DOPE) and cholesterol (CHOL) were purchased from Avanti Polar Lipids Inc. (Alabaster, AL, USA). Amphotericin B (AmB) was obtained from Duchefa Biochemical Co. (The Netherlands). As a control transfection reagent, Lipofectamine ${ }^{\circledR}$ was purchased from Invitrogen (San Diego, CA, USA). All other chemicals were of reagent grade and used without further purification.

\section{Purification of Plasmid DNA}

The plasmid DNA was amplified in the Escherichia coli DH5 $\alpha$ and purified with the Qiagen Plasmid Mega kit (Qiagen, CA, USA), according to the manufacturer's instruction. The integrity of DNA preparation was confirmed on a $1 \%$ agarose gel.

\section{Preparation of Cationic Liposomes and Liposomes/ Plasmid DNA Complexes}

Cationic liposomes were prepared by lipid film method. Stock solution of each lipid were mixed at the molar ratio of DOTAP/DOPE/CHOL $=5: 4: 1$ in chloroform. ${ }^{16}$ ) The organic phase was removed at $50^{\circ} \mathrm{C}$ on a rotary evaporator (KU-NLW, Sunil Instrument Co., Korea). The dried lipid film was flushed with nitrogen gas to remove traces of organic solvent and hydrated with phosphate-buffered saline (PBS). After sonication at about $30^{\circ} \mathrm{C}$ for $30 \mathrm{~min}$, the solution was extruded 10 times through a $100-\mathrm{nm}$ polycarbonate membrane using an extruder (Northern Lipids Inc., Canada). The cationic liposomes containing $\mathrm{AmB}$ were prepared at a ratio of lipid solution: $\mathrm{AmB}=4: 1$ similar to process of blank liposomes. ${ }^{16)}$ The complexes were prepared by mixing DNA and cationic liposomes at the various ratios of cationic lipid to plasmid DNA $(4-40, w / w)$. Then, the complexes were incubated for $15 \mathrm{~min}$ at $37^{\circ} \mathrm{C}$ to facilitate complex formation.

\section{Measurement of Particle Size and Zeta Potential}

Particle size distribution of cationic liposomes with or without plasmid DNA was determined by light scattering spectrophotometer (ELS-8000, Otsuka Electronics Co., Japan). The samples were diluted with deionized water, and then transferred into a quartz cuvette in an ELS-8000 dynamic light scattering instrument. Zeta potential of cationic liposomes and complexes was measured by electrophoretic light scattering spectrophotometer. Data were analyzed using a software package (ELS-8000 software) supplied by the manufacturer.

\section{Agarose Gel Retardation}

Complex formation between the cationic liposomes and plasmid DNA was assessed using an agarose gel electrophoresis. ${ }^{17)}$ Various ratios $(4-40, \mathrm{w} / \mathrm{w})$ of cationic lipid/DNA mixtures with fixed amount of plasmid DNA $(1 \mu \mathrm{g})$ were loaded onto a $1 \%$ agarose gel. As a control, Lipofectamine ${ }^{\circledR /}$ DNA complex containing $0.5 \mathrm{mg}$ of plasmid DNA at the optimal ratio of 6 (Lipofectamine ${ }^{\circledR}$ to DNA, w/w) was also loaded on the gel. Gel electrophoresis was carried out in a TAE buffer (40 mM Tris-acetate, $1 \mathrm{mM}$ EDTA) at $50 \mathrm{mV}$. Ethidium bromide stained DNA was visualized using a UV. This gel retardation experimental was repeated using AmB-containing liposomes.

\section{Complex Stability against DNase I Digestion}

The cationic liposomes were mixed with $0.2 \mu \mathrm{g}$ of plasmid DNA at the ratio of 40 (DOTAP to DNA, w/w) and allowed to form complexes for $15 \mathrm{~min}$ at room temperature and $37^{\circ} \mathrm{C}$. For DNase I digestion, the cationic liposomes were prepared in water instead of PBS to avoid the interfering effect of high salt on DNase I activity. ${ }^{17)}$ Naked DNA $(0.2 \mu \mathrm{g})$ or the cationic liposomes/DNA complex containing the same amount of DNA was exposed to DNase I for $30 \mathrm{~min}$ at $37^{\circ} \mathrm{C}$ to investigate the stability of the complex against nuclease digestion. DNA was retrieved by phenol/chloroform extraction followed by ethanol precipitation and visualized on an agarose gel containing ethidium bromide.

\section{Results and Discussion}

\section{Particle Size and Zeta Potential}

In order to investigate the effect of drug loading on particle size and zeta potential, we determined the particle size and zeta potential of blank liposomes or drug-loaded liposomes. Particle size of drug free liposomes and drug loaded liposomes were $260.9 \mathrm{~nm}$ and $244.5 \mathrm{~nm}$, respectively (Table I). Zeta potentials of liposomes were $70.03 \mathrm{mV}$ for drug free liposomes and $58.18 \mathrm{mV}$ for drug loaded liposomes (Table I). The particle size and zeta potential of complexes were also determined by varying drug loading. After drug loading, the size of complexes was increased, but their zeta potential was not changed much (Figure 1). Higher ratio of liposomes to DNA resulted in smaller particle size irrespective of drug loading (Figure 1a). However, the particle size of complexes between drug loaded liposomes/plasmid DNA was larger than that of drug free complexes. Naturally, zeta potential had a tendency to increase according to the ratio of liposomes to plasmid DNA; the amount of DNA was fixed as $1 \mu \mathrm{g}$ and the content 
Table I-Physicochemical Properties of Blank Liposomes and Drug loaded Liposomes

\begin{tabular}{ccc}
\hline \hline Liposomes & Particle size $(\mathrm{nm})$ & Zeta potential $(\mathrm{mV})$ \\
\hline Blank & 260.9 & 70.03 \\
Drug loaded & 244.5 & 58.18
\end{tabular}

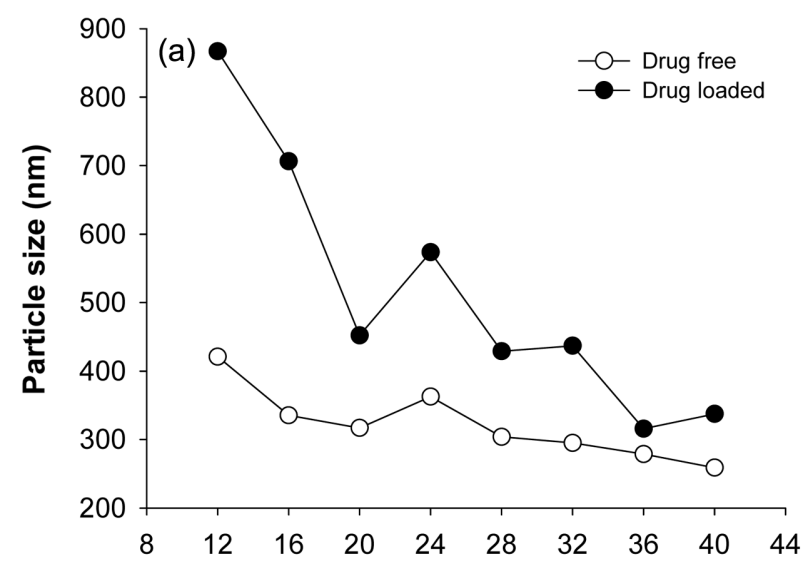

Ratio of cationic lipid to DNA (w/w)

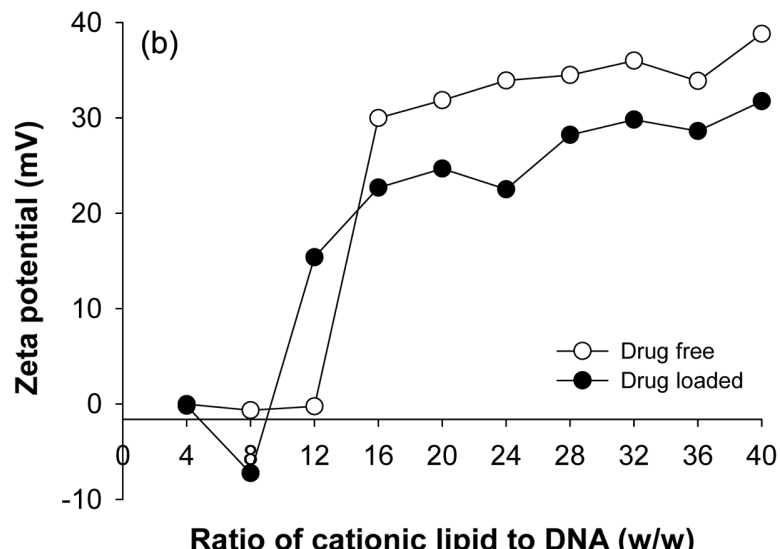

Figure 1-Physicochemical characterization of complexes. (a) Particle size of drug free complexes and drug loaded complexes. (b) Zeta potential of drug free complexes and drug loaded complexes.

of cationic lipid, DOTAP, was increased (Figure 1b). In a similar manner, the size of doxorubicin (DOX)-loaded micelle/ DNA complexes was also increased when compared to blank peptide micelle/DNA complexes; and as expected, their zeta potential was similar, since both DOX-loaded micelles and blank micelles had a similar zeta potential. ${ }^{15)}$ In particular, the size of DOX-loaded FA32 micelle/DNA complexes was below $200 \mathrm{~nm}$ at N/P 15 or above, and at these N/P ratios, their zeta potential was around $20 \mathrm{mV}$. The small size and net positive surface charge of AmB-loaded liposomes may provide desir- (a)
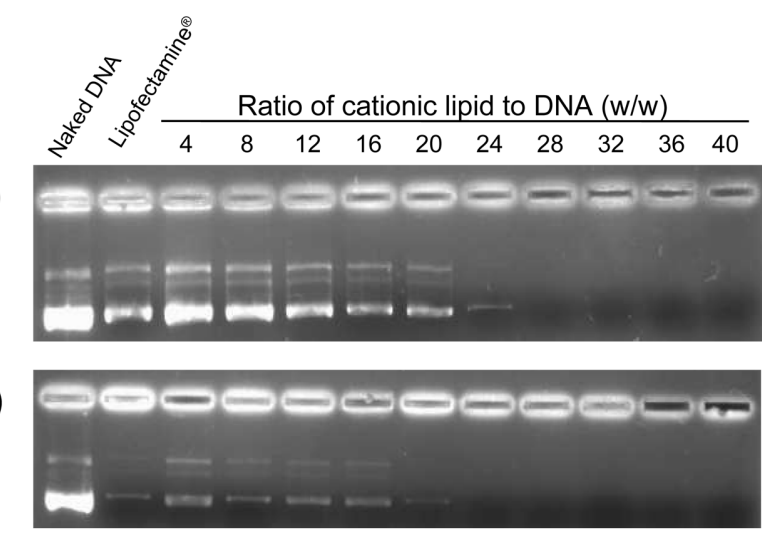

Figure 2-Agarose gel retardation of (a) drug free complexes and (b) drug loaded complexes.

able properties for easy cellular uptake and gene/drug delivery like DOX-loaded micelle. ${ }^{15}$ )

\section{Gel Retardation Assay}

The DNA binding ability of cationic liposomes was studied through gel retardation assay. ${ }^{17)}$ As shown in Figure 2a, free DNA that failed to form complex with cationic liposomes was clearly visualized at complex ratios of 4-24 (w/w). However, free DNA disappeared when the ratio of DOTAP to DNA was above $28(\mathrm{w} / \mathrm{w})$ (Figure 2a). At the ratios over 28, cationic liposomes were able to bind DNA efficiently, and the complete retardation of DNA mobility was achieved at ratio 28 . Complexes formed at under ratios 24 shows incomplete complexation (Figure 2a). In addition, the gel retardation assays from the DNA complexes formed with drug-loaded liposomes also yielded a similar retardation profile (Figure 2b, ratios over 24), although AmB-loaded liposomes had lower zeta potential values than the drug free liposomes (Figure 1b). However, DOX-loaded micelles had a similar zeta potential value when compared to the corresponding blank liposomes and showed same gel retardation at various $\mathrm{N} / \mathrm{P}$ ratios of cationic micelles to plasmid DNA. $^{15)}$

\section{Stability of the Liposomes/DNA Complex}

We also examined whether DNA in the complex could be protected from DNase I digestion, ${ }^{17)}$ since complexes are not able to work in vivo owing to the interrupting effect of nuclease. Naked DNA was completely digested by 0.25 units of DNase I (Figure 3). However, the complexed DNA was protected from DNase I independent on incubation temperature, such as room temperature and $37^{\circ} \mathrm{C}$. When incubating plasmid DNA with cationic liposomes at $37^{\circ} \mathrm{C}$, the complexes were firmly complexed compared with those incubated at 

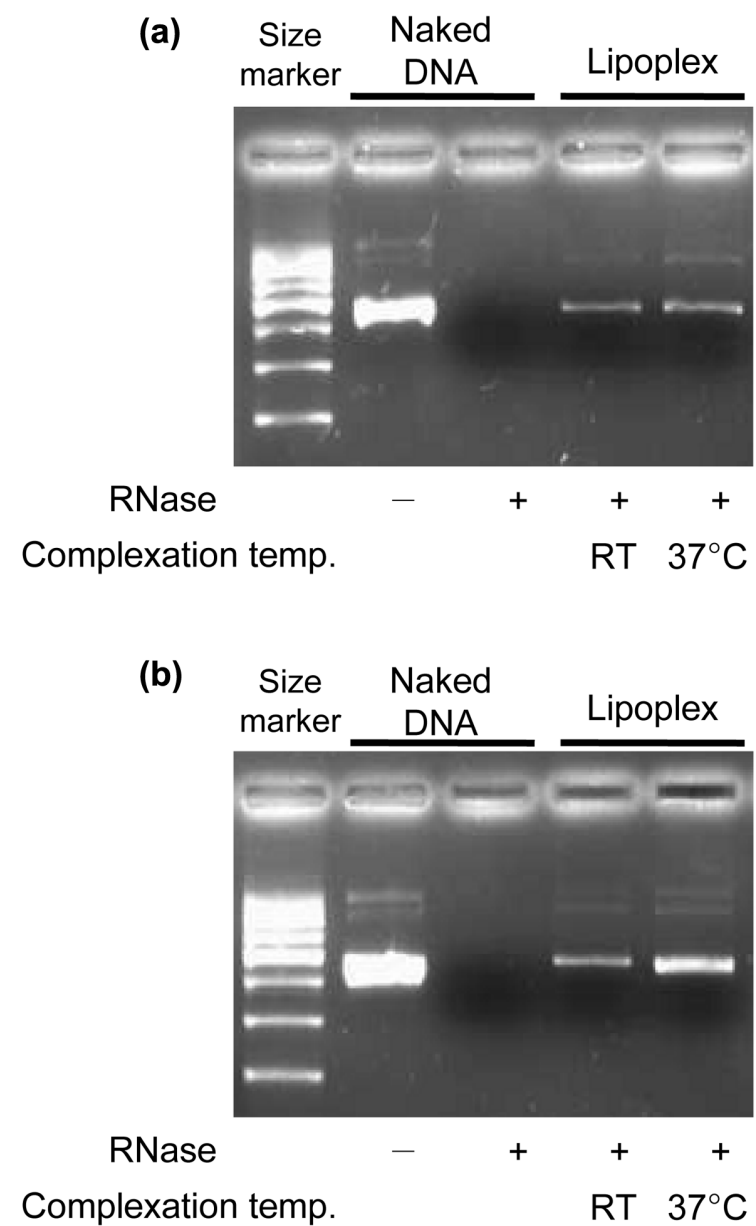

Figure 3-Stability for DNase of plasmid DNA by (a) drug free complexes and (b) drug loaded complexes.

room temperature. Moreover, there was no effect of drug loading on the protection from DNase I (Figure 3b).

\section{Conclusion}

These results demonstrated that the complex formation of cationic liposomes and plasmid DNA was not influenced by drug loading. Moreover, drug loading did not affect the stability of complexed DNA against DNase I. Further studies are needed to investigate the effect of incubating temperature on the formation of complexes and to determine the effect of drug loading on cellular uptake and in vivo fate of complexes.

\section{Acknowledgements}

This work was supported by the National Research Foundation (NRF) grant (NRF-2006-003-E00439) funded by the Korea government (Ministry of Education, Science and Technology).

\section{References}

1) X. Gao, K.S. Kim and D. Liu, Nonviral gene delivery: what we know and what is next, AAPS J., 9, E92-104 (2007).

2) W.J. Choi and C.K. Kim, Recent advances and future strategy in gene delivery system, J. Kor. Pharm. Sci., 30, 1-12 (2000).

3) H. Joensuu, K. Holli, M. Heikkinen, E. Suonio, A.R. Aro, P. Hietanen and R. Huovinen, Combination chemotherapy versus single-agent therapy as first- and second-line treatment in metastatic breast cancer: a prospective randomized trial, J. Clin. Oncol., 16, 3720-3730 (1998).

4) J.O. Shaughnessy, D. Miles, S. Vukelja, V. Moiseyenko, J.P. Ayoub, G. Cervantes, P. Fumoleau, S. Jones, W.Y. Lui, L. Mauriac, C. Twelves, G. van Hazel, S. Verma and R. Leonard, Superior survival with capecitabine plus docetaxel combination therapy in anthracycline-pretreated patients with advanced breast cancer: phase III trial results, J. Clin. Oncol., 20, 28122823 (2002).

5) J.D. McConnel, C.G. Roehrborn, O.M. Bautista, G.L. Andriole, C.M. Dixon and J.W. Kusek, H. Lepor, K.T. McVary, L.M. Nyberg Jr., H.S. Clarke, E.D. Crawford, A. Diokno, J.P. Foley, H.E. Foster, S.C. Jacobs, S.A. Kaplan, K.J. Kreder, M.M. Lieber, M.S. Lucia, G.J. Miller, M. Menon, D.F. Milam, J.W. Ramsdell, N.S. Schenkman, K.M. Slawin and J.A. Smith, The long-term effect of doxazosin, finasteride, combination therapy on the clinical progression of benign prostatic hyperplasia, New Engl. J. Med., 349, 2387-2398 (2003).

6) P. Duesberg, R. Li, R. Sachs, A. Fabarius, M. Upender and R. Hehlmann, Cancer drug resistance: the central role of the karyotype, Drug Resist. Updat., 10, 51-58 (2003).

7) R. Li, R. Hehlman, R. Sachs and P. Duesberg, Chromosomal alterations cause the high rates and wide ranges of drug resistance in cancer cells, Cancer Genet. Cytogenet., 163, 44-56 (2003).

8) R.A. Gjerset and D. Mercola, Sensitization of tumors to chemotherapy through gene therapy, Adv. Exp. Med. Biol., 465, 273-291 (2000).

9) B.D. Lee, K.J. French, Y. Zhuang and C.D. Smith, Development of a syngeneic in vivo tumor model and its use in evaluating a novel P-glycoprotein modulator, PGP-4008, Oncol. Res., 14, 49-60 (2003).

10) R. Koivusalo, E. Krausz, H. Helenius and S. Hietanen, Chemotherapy compounds in cervical cancer cells primed by reconstitution of $\mathrm{p} 53$ function after short interfering RNA mediated degradation of human Papilloma virus 18 E6 mRNA: opposite effect of siRNA in combination with different drugs, Mol. Pharmacol., 68, 372-382 (2005).

11) R.R. Nair, J.R. Rodgers and L.A. Schwarz, Enhancement of transgene expression by combining glucocorticoids and antimitotic agents during transient transfection using DNA-cationic liposomes, Mol. Ther, 5, 455-462 (2002).

12) F. Liu, L.M. Shollenberger and L. Huang, Non-immunostimulatory nonviral vectors, FASEB J., 18, 1779-1781 (2004).

13) Y. Wang, S. Gao, W.H. Ye, H.S. Yoon, Y.Y. Yang, Co-delivery 
of drugs and DNA from cationic core-shell nanoparticles selfassembled from a biodegradable copolymer, Nat. Mater., 5, $791-796$ (2006).

14) Y. Wang, L.S. Wang, S.H. Goh and Y.Y. Yang, Synthesis and characterization of cationic micelle self-assembled from a biodegradable copolymer for gene delivery, Biomacromolecules, 8, 1028-1037 (2007).

15) N. Wiradharma, Y.W. Tong and Y.Y. Yang, Self-assembled oligopeptide nanostructures for co-delivery of drug and gene with synergistic therapeutic effect, Biomaterials, 30, 3100-3109 (2009).

16) J.W. Kang, E. Davaa, Y.T. Kim and J.S. Park, A new vaginal delivery system based on a dispersion of cationic liposomes in a thermosensitive gel for the controlled release of amphotericin B, J. Drug Target., submitted (2009).

17) K.A. Min, S.K. Lee and C.K. Kim, Improved gene expression pattern using Epstein-Barr virus (EBV)-based plasmid and cationic emulsion, Biomaterials, 26, 1063-1070 (2004). 